\title{
On iterative solutions of a split feasibility problem with nonexpansive mappings
}

\author{
M.A. Kutbi ${ }^{1}$, A. Latif ${ }^{*}$ (D) and X. Qin ${ }^{2}$
}

\footnotetext{
"Correspondence: alatif@kau.edu.sa ${ }^{1}$ Department of Mathematics, King Abdulaziz University, Jeddah, Saudi Arabia

Full list of author information is available at the end of the article
}

\begin{abstract}
We analyze iterative solutions of a split feasibility problem with common fixed point constraints of a family of nonexpansive mappings. We present solution theorems of the feasibility problem under some weak assumptions imposed on different mappings and control sequences.
\end{abstract}

MSC: 47H05; 47H09; 47J20; 65K15

Keywords: Fixed Point; Hilbert space; Monotone operator; Nonexpansive operator; Variational inequality

\section{Introduction-preliminaries}

Let $H_{1}$ and $H_{2}$ be Hilbert spaces, and let $C$ and $Q$ be nonempty convex closed sets in $H_{1}$ and $H_{2}$, respectively. Let $A: H_{1} \rightarrow H_{2}$ be a bounded linear mapping.

In 1994, Censor and Elfving [10] introduced the well-known split feasibility problem for modeling inverse problems formulated as follows:

$$
\text { Find } x^{*} \in C \text { such that } A x^{*} \in Q \text {. }
$$

It can be formulated as the following convex feasibility problem:

$$
\text { Find } x^{*} \in C \cap A^{-1}(Q) \text {. }
$$

Both split feasibility and convex feasibility problems are much related to a number of realworld applications, for example, signal processing, intensity-modulated radiation therapy, and image reconstruction; see $[9,11,35]$ and the references therein. Recently, a number of regularized iterative methods have been introduced and investigated for solutions of the feasibility problems in either Banach or Hilbert spaces by many authors; see $[1-5,16,17$, $19,28,31$ ] and the references therein.

Let $H$ be a real Hilbert space endowed with inner product $\langle\cdot, \cdot\rangle$ and induced norm $\|\cdot\|$. Let $S$ be a mapping on $H$. Fix $(S)$ stands for a fixed point set of $S$. Recall that $S$ is said to be nonexpansive if

$$
\|S x-S y\| \leq\|x-y\|, \quad \forall x, y \in H .
$$

(c) The Author(s) 2019. This article is distributed under the terms of the Creative Commons Attribution 4.0 International License (http://creativecommons.org/licenses/by/4.0/), which permits unrestricted use, distribution, and reproduction in any medium, provided you give appropriate credit to the original author(s) and the source, provide a link to the Creative Commons license, and indicate if changes were made. 
It is well known that every nonexpansive mapping satisfies the following property:

$$
2\langle S x-S y,(y-S y)-(x-S x)\rangle \leq\|(x-S x)-(y-S y)\|^{2}, \quad \forall x, y \in H .
$$

The mapping $S$ is said to be quasinonexpansive if

$$
\|x-S y\| \leq\|x-y\|, \quad \forall x \in \operatorname{Fix}(S) \neq \emptyset, y \in H .
$$

It is obvious that quasinonexpansive mappings may not be continuous beyond their fixedpoint sets. Every quasinonexpansive mapping $S$ satisfies the following property:

$$
2\langle x-S y,(y-S y)\rangle \leq\|y-S y\|^{2}, \quad \forall x \in \operatorname{Fix}(S) \neq \emptyset, y \in H .
$$

It is said to be firmly nonexpansive if

$$
\|S x-S y\|^{2} \leq\langle S x-S y, x-y\rangle, \quad \forall x, y \in H .
$$

It is is said to be firmly quasinonexpansive if

$$
\|x-S y\|^{2} \leq\langle x-S y, x-y\rangle, \quad \forall x \in \operatorname{Fix}(S) \neq \emptyset, y \in H .
$$

It is is said to be contractive if there exists a constant $\kappa \in(0,1)$ such that

$$
\|S x-S y\|^{2} \leq \kappa\|x-y\|, \quad \forall x, y \in H
$$

Contractive mappings and their extensions are important classes of nonlinear mappings since they are connected with differential equations and nonsmooth optimization; see [7, $8,14,21]$ and the references therein. Recently, they have been extensively analyzed via projection-based iterative methods. It deserves mentioning that the methods based on nearest-point projections are not efficient from the viewpoint of numerical computation. Let $\operatorname{Proj}_{C}^{H}$ be the nearest-point (metric) projection from $H$ onto $C$, that is,

$$
\operatorname{Proj}_{C}^{H} y:=\left\{x \in C:\|x-y\|=\operatorname{dist}_{C}(y)\right\},
$$

where $\operatorname{dist}_{C}(y):=\inf _{x \in C}\|x-y\|$ for $y \in H$.

To avoid using nearest projections, Yamada [33] recently studied a descent method, which is known as the Yamada descent algorithm. This algorithm is as follows:

$$
u_{n+1}=\left(I-\alpha_{n+1} \mu F\right) T u_{n}, \quad \forall n \in \mathbb{N},
$$

where $\left\{\alpha_{n}\right\}$ is a real sequence in $(0,1), \mu$ is some positive real number, $T$ is a nonexpansive mapping on $H$, and $F$ is $\eta$-strongly monotone and $\mathcal{L}$-Lipschitz continuous on $H$. Recently, many authors studied the Yamada descent methods for nonexpansive nonlinear operators in Banach or Hilbert spaces; see [13, 22, 23, 26] and the references therein. 
Now we recall some useful notions. Let $F: C \rightarrow H$ be a nonself single-valued operator. It is called

(i) monotone if

$$
\left\langle x^{*}-x, F x^{*}-F x\right\rangle \geq 0, \quad \forall x^{*}, x \in C
$$

(ii) strongly monotone if there exists a positive constant $\eta>0$ such that

$$
\eta\left\|x^{*}-x\right\|^{2} \leq\left\langle x^{*}-x, F x^{*}-F x\right\rangle, \quad \forall x^{*}, x \in C .
$$

(iii) $\mathcal{L}$-Lipschitz if there exists $\mathcal{L}>0$ such that

$$
\left\|F x-F x^{*}\right\| \leq \mathcal{L}\left\|x-x^{*}\right\|, \quad \forall x^{*}, x \in C .
$$

Let $M: H \rightarrow 2^{H}$ be a set-valued monotone mapping. The zero-point set of $M$ is denoted by $M^{-1}(0)$. Recall that $M$ is said to be monotone if, for all $x, y \in H, u \in M x$, and $v \in M y$

$$
\langle x-y, u-v\rangle \geq 0
$$

It is said to be maximal if its graph $\operatorname{Graph}(M)$ is not properly contained in the graph of any other monotone mapping. If $M$ is maximally monotone, then $\operatorname{Graph}(M)$ is weakly strongly closed; see [24] and the references therein. A well-known fact is that for $(x, u) \in H \times H$, $\langle x-y, u-v\rangle \geq 0$ for all $(y, v) \in \operatorname{Graph}(M)$ implies that $u \in M(x)$ iff $M$ is maximal. Let $N$ be a maximal monotone operator with domain $\operatorname{Dom}(N)$ and range $H$. Define the mapping $\operatorname{Res}_{\lambda}^{N}: H \rightarrow \operatorname{Dom}(M)$ associated with index $\lambda$ by

$$
\operatorname{Res}_{\lambda}^{N} x=(\lambda N+\mathrm{Id})^{-1}(x), \quad \forall x \in H
$$

where Id is the identity operator on $H$. If $N$ is the subdifferential of proper convex lower semicontinuous functions, then the resolvent operator is the known proximity operator. The resolve operator plays a significant role in nonsmooth optimization problems. A variety of nonlinear problems, including variational inequalities and equilibrium problems, can be formulated as finding a zero of a maximal monotone operator. It is known that $\operatorname{Fix}\left(\operatorname{Res}_{\lambda}^{N}\right)=N^{-1}(0)$; see $[15,18,20,27,34]$ and the references therein.

Let $N$ be a set-valued maximal monotone operator on $H_{1}$, and let $M$ be a set-valued maximal monotone operator on $\mathrm{H}_{2}$. We consider the following split inclusion problem: find $x^{*} \in H_{1}$ such that

$$
0 \in N\left(x^{*}\right), y^{*}=A x^{*} \in H_{2} \quad \text { solves } \quad 0 \in M\left(y^{*}\right),
$$

where $A$ is a linear bounded mapping from $H_{1}$ to $H_{2}$. We denote by $\operatorname{SIP}(M, N)$ the solution set of problem (1.3).

In this paper, we analyze iterative solutions of a split feasibility problem with common fixed-point constraints of a family of nonexpansive mappings. We present solution theo- 
rems of the feasibility problem under some weak assumptions imposed on different mappings. For our main result, we also need the following tools.

Let $S_{i}$ be a nonexpansive mapping on $C$, and let $\eta_{i}$ be real numbers with $0<\eta_{i}<1$ for each $i \geq 1$. Let $W_{n}$ be a mapping on $C$ defined for each $n \geq 1$ by

$$
\begin{aligned}
& U_{n, n+1}=I, \\
& U_{n, n}=\left(1-\eta_{n}\right) I+\eta_{n} S_{n} U_{n, n+1}, \\
& U_{n, n-1}=\left(1-\eta_{n-1}\right) I+\eta_{n-1} S_{n-1} U_{n, n}, \\
& \vdots \\
& U_{n, k}=\left(1-\eta_{k}\right) I+\eta_{k} S_{k} U_{n, k+1}, \\
& U_{n, k-1}=\left(1-\eta_{k-1}\right) I+\eta_{k-1} S_{k-1} U_{n, k}, \\
& \vdots \\
& U_{n, 2}=\left(1-\eta_{2}\right) I+\eta_{2} S_{2} U_{n, 3}, \\
& U_{n, 1}=\left(1-\eta_{1}\right) I+\eta_{1} S_{1} U_{n, 2}, \\
& W_{n}=U_{n, 1} .
\end{aligned}
$$

It is clear that $W_{n}: C \rightarrow C$, governed by $S_{1}, S_{2}, \ldots, S_{n}$ and $\eta_{1}, \eta_{2}, \ldots, \eta_{n}$, is a nonexpansive mapping; see [29] and the references therein. We further assume that $0<\eta_{i} \leq \eta<1$ for $i \geq 1$, where $\eta$ is a constant in $(0,1)$.

Lemma 1.1 ([29]) Let $C$ be a convex and closed set in a Hilbert space $H$, and let $S_{i}$ be nonexpansive mappings on $C$ with fixed points. If $\bigcap_{i=1}^{\infty} \operatorname{Fix}\left(S_{i}\right) \neq \emptyset$, then

(1) $\lim _{n \rightarrow \infty} U_{n, k}$ exists for each positive integer $k$ and each $x \in C$;

(2) the mapping $W: C \rightarrow C$ defined by

$$
W x:=\lim _{n \rightarrow \infty} W_{n} x=\lim _{n \rightarrow \infty} U_{n, 1} x, \quad x \in C,
$$

is a nonexpansive mapping with $\operatorname{Fix}(W)=\bigcap_{i=1}^{\infty} \operatorname{Fix}\left(S_{i}\right)=\operatorname{Fix}\left(W_{n}\right)$.

Lemma 1.2 ([12]) Let $C$ be a convex and closed set in a Hilbert space $H$, and let $S_{i}$ be a nonexpansive mappings on $C$ with fixed points. Assume that $\bigcap_{i=1}^{\infty} F\left(S_{i}\right) \neq \emptyset$. Then $\lim _{n \rightarrow \infty} \sup _{x \in K}\left\|W_{n} x-W x\right\|=0$ for any bounded set $K \subset C$.

Lemma 1.3 ([33]) Let $H$ be a Hilbert space. Let $F$ be an $\mathcal{L}$-Lipschitz continuous and $\eta$ strongly monotone mapping on the space $H$. Let $T^{\alpha}$ be a mapping on the space $H$ defined by $T^{\alpha} x=x-\mu \alpha F x$ for $x \in H$, where $\alpha$ is a real number in $(0,1)$. If $0<\mathcal{L}^{2} \mu \in(0,2 \eta)$ and $\tau=1-\sqrt{1-\mu\left(2 \eta-\mu \mathcal{L}^{2}\right)} \in(0,1]$, then

$$
\left\|T^{\alpha} x-T^{\alpha} y\right\| \leq(1-\tau \alpha)\|x-y\|, \quad \forall x, y \in H
$$

Lemma 1.4 ([32]) Let $\left\{\alpha_{n}\right\},\left\{\beta_{n}\right\}$, and $\left\{\gamma_{n}\right\}$ be sequences of real numbers such that $\alpha_{n} \in$ $[0,1], \sum_{n=1}^{\infty} \alpha_{n}=\infty, \lim _{\sup _{n \rightarrow \infty}} \beta_{n} \leq 0$, and $\sum_{n=1}^{\infty} \gamma_{n}<\infty$ Let $\left\{\lambda_{n}\right\}$ be a sequence of non- 
negative real numbers such that

$$
\lambda_{n+1} \leq\left(1-\alpha_{n}\right) \lambda_{n}+\alpha_{n} \beta_{n}+\gamma_{n} .
$$

Then $\lim _{n \rightarrow \infty} \lambda_{n}=0$.

Lemma 1.5 ([25]) Let $\left\{x_{n}\right\}$ be a sequence in a real Hilbert space $H$. If $x_{n} \rightarrow x$, then

$$
\liminf _{n \rightarrow \infty}\left\|x_{n}-x\right\|<\liminf _{n \rightarrow \infty}\left\|x_{n}-y\right\|
$$

for any $y \in X$ with $y \neq x$. This is also equivalent to

$$
\limsup _{n \rightarrow \infty}\left\|x_{n}-x\right\|<\limsup _{n \rightarrow \infty}\left\|x_{n}-y\right\|
$$

Lemma 1.6 ([6, resolvent equality]) Let $H$ be a Hilbert space. Let $N$ be a set-valued maximal operator on $H$. For parameters $\lambda>0$ and $\mu>0$, we have

$$
\operatorname{Res}_{\mu}^{N}\left(\left(1-\frac{\mu}{\lambda}\right) \operatorname{Res}_{\lambda}^{N} x+\frac{\mu}{\lambda} x\right)=\operatorname{Res}_{\lambda}^{N} x, \quad \forall x \in H .
$$

Lemma 1.7 ([30]) Let $H$ be a Hilbert space. Let $\left\{x_{n}\right\}$ and $\left\{y_{n}\right\}$ be bounded sequences in $H$ with $x_{n+1}=\left(1-\beta_{n}\right) y_{n}+\beta_{n} x_{n}$ for all $n \geq 0$ and

$$
\limsup _{n \rightarrow \infty}\left(\left\|y_{n+1}-y_{n}\right\|-\left\|x_{n+1}-x_{n}\right\|\right) \leq 0,
$$

where $\left\{\beta_{n}\right\}$ is a sequence in $(0,1)$ such that $\liminf _{n \rightarrow \infty} \beta_{n}>0$ and $\lim \sup _{n \rightarrow \infty} \beta_{n}<1$. Then $\lim _{n \rightarrow \infty}\left\|y_{n}-x_{n}\right\|=0$.

\section{Main results}

Theorem 2.1 Let $H_{1}$ and $H_{2}$ be Hilbert spaces, and let $N$ and $M$ be set-valued maximal monotone mappings on $H_{1}$ and $H_{2}$, respectively. Let $S_{i}$ be nonexpansive mappings on $H_{1}$ for all integers $i \geq 1$. Let $F: H_{1} \rightarrow H_{1}$ be an $\mathcal{L}$-Lipschitz continuous and $\tau$-strongly monotone mapping. Let $A$ be a linear bounded operator from $H_{1}$ to $H_{2}$, and let $A^{*}$ be its adjoint operator. Assume that $\bigcap_{i=1}^{\infty} \operatorname{Fix}\left(S_{i}\right) \cap \operatorname{SIP}(M, N) \neq \emptyset$. Let $\left\{x_{n}\right\}$ be a vector sequence in $H_{1}$ generated by the iterative process

$$
\left\{\begin{array}{l}
x_{1} \in H_{1} \\
y_{n}=\gamma_{n} \operatorname{Res}_{s_{n}}^{N}\left(x_{n}+\gamma A^{*}\left(\operatorname{Res}_{r_{n}}^{M}-I\right) A x_{n}\right)+\left(1-\gamma_{n}\right) x_{n}, \\
x_{n+1}=\beta_{n}\left(I-\mu \alpha_{n} F\right) W_{n} y_{n}+\left(1-\beta_{n}\right) x_{n}, \quad n \geq 1
\end{array}\right.
$$

where $\gamma$ and $\mu$ are two positive real numbers, $\left\{s_{n}\right\}$ and $\left\{r_{n}\right\}$ are two positive real number sequences, $\left\{\alpha_{n}\right\},\left\{\beta_{n}\right\}$, and $\left\{\gamma_{n}\right\}$ are real number sequences in $(0,1)$. Suppose that $\gamma \in\left(0, \frac{1}{\|A\|^{2}}\right)$, $\mu \in\left(0, \frac{2 \tau}{\mathcal{L}^{2}}\right), \liminf _{n \rightarrow \infty} s_{n}>0, \lim _{n \rightarrow \infty}\left|s_{n}-s_{n+1}\right|<\infty, \liminf _{n \rightarrow \infty} r_{n}>0, \lim _{n \rightarrow \infty}\left|r_{n}-r_{n+1}\right|<$ $\infty, \sum_{n=1}^{\infty} \alpha_{n}=\infty,\left\{\beta_{n}\right\}$ is number sequence in $\left[\bar{\beta}, \bar{\beta}^{\prime}\right]$, where $\bar{\beta}$ and $\bar{\beta}^{\prime}$ are two real numbers in $(0,1)$, such that $\lim _{n \rightarrow \infty}\left|\beta_{n+1}-\beta_{n}\right|=0$, and $\left\{\gamma_{n}\right\}$ is a sequence in $[\bar{\gamma}, 1]$, where $\bar{\gamma} \in(0,1]$, 
such that $\lim _{n \rightarrow \infty}\left|\gamma_{n+1}-\gamma_{n}\right|=0$. Then the sequence $\left\{x_{n}\right\}$ converges strongly to $\tilde{x} \in H_{1}$, which is a unique solution of the variational inequality

$$
\langle\widetilde{x}-y, F \widetilde{x}\rangle \leq 0, \quad \forall y \in \bigcap_{i=1}^{\infty} \operatorname{Fix}\left(S_{i}\right) \cap \operatorname{SIP}(M, N) .
$$

Proof The proof is split into four steps.

Step 1. We prove that $\left\{x_{n}\right\}$ is a bounded vector sequence in $H_{1}$.

For any fixed $p \in \bigcap_{i=1}^{\infty} \operatorname{Fix}\left(S_{i}\right) \cap \operatorname{SIP}(M, N)$, we conclude $A p=\operatorname{Res}_{r_{n}}^{M} A p, p=\operatorname{Res}_{s_{n}}^{N} p$, and $p=S_{i} p$ for each $i \geq 1$. Since $A p$ is a fixed point of $\operatorname{Res}_{r_{n}}^{M}$ and $\operatorname{Res}_{r_{n}}^{M}$ is a (firmly) nonexpansive mapping, we have

$$
\left\langle\left(\operatorname{Res}_{r_{n}}^{M}-I\right) A x_{n}, \operatorname{Res}_{r_{n}}^{M} A x_{n}-A p\right\rangle \leq \frac{\left\|\operatorname{Res}_{r_{n}}^{M} A x_{n}-A x_{n}\right\|^{2}}{2} .
$$

Putting

$$
z_{n}=\operatorname{Res}_{s_{n}}^{N}\left(x_{n}+\gamma A^{*}\left(\operatorname{Res}_{r_{n}}^{M}-I\right) A x_{n}\right),
$$

(2.1) sends us to

$$
\begin{aligned}
\| z_{n}- & p \|^{2} \\
\leq & \left\|\gamma A^{*}\left(\operatorname{Res}_{r_{n}}^{M}-I\right) A x_{n}+\left(x_{n}-p\right)\right\|^{2} \\
\leq & \gamma^{2}\|A\|^{2}\left\|\left(\operatorname{Res}_{r_{n}}^{M}-I\right) A x_{n}\right\|^{2}+2 \gamma\left\langle A^{*}\left(\operatorname{Res}_{r_{n}}^{M}-I\right) A x_{n}, x_{n}-p\right\rangle+\left\|x_{n}-p\right\|^{2} \\
= & \gamma\left(\gamma\|A\|^{2}-2\right)\left\|\left(\operatorname{Res}_{r_{n}}^{M}-I\right) A x_{n}\right\|^{2} \\
& +2 \gamma\left(\left(\operatorname{Res}_{r_{n}}^{M}-I\right) A x_{n}, \operatorname{Res}_{r_{n}}^{M} A x_{n}-A p\right\rangle+\left\|x_{n}-p\right\|^{2} \\
\leq & \gamma\left(\gamma\|A\|^{2}-1\right)\left\|\left(\operatorname{Res}_{r_{n}}^{M}-I\right) A x_{n}\right\|^{2}+\left\|x_{n}-p\right\|^{2},
\end{aligned}
$$

which leads to

$$
\begin{aligned}
\left\|y_{n}-p\right\|^{2} & \leq \gamma_{n}\left\|z_{n}-p\right\|^{2}+\left(1-\gamma_{n}\right)\left\|x_{n}-p\right\|^{2} \\
& \left.\leq\left\|x_{n}-p\right\|^{2}-\gamma_{n} \gamma\left(1-\gamma\|A\|^{2}\right)\left\|\left(\operatorname{Res}_{r_{n}}^{M}-I\right) A x_{n}\right\|^{2}\right) .
\end{aligned}
$$

The restriction imposed on parameter $\gamma$ tells us that $\left\|y_{n}-p\right\| \leq\left\|x_{n}-p\right\|$. Since $W_{n}$ is a nonexpansive mapping for each $n$, we find from Lemma 1.3 that

$$
\begin{aligned}
\left\|x_{n+1}-p\right\| & \leq \beta_{n}\left\|\left(I-\mu \alpha_{n} F\right) W_{n} y_{n}-\left(I-\mu \alpha_{n} F\right) p-\mu \alpha_{n} F p\right\|+\left(1-\beta_{n}\right)\left\|x_{n}-p\right\| \\
& \leq \beta_{n}\left\|\left(I-\mu \alpha_{n} F\right) W_{n} y_{n}-\left(I-\mu \alpha_{n} F\right) p\right\|+\mu \beta_{n} \alpha_{n}\|F p\|+\left(1-\beta_{n}\right)\left\|x_{n}-p\right\| \\
& \leq \beta_{n}\left(1-\tau \alpha_{n}\right)\left\|W_{n} y_{n}-W_{n} p\right\|+\mu \beta_{n} \alpha_{n}\|F p\|+\left(1-\beta_{n}\right)\left\|x_{n}-p\right\| \\
& \leq \beta_{n}\left(1-\tau \alpha_{n}\right)\left\|y_{n}-p\right\|+\mu \beta_{n} \alpha_{n}\|F p\|+\left(1-\beta_{n}\right)\left\|x_{n}-p\right\| \\
& \leq \tau \alpha_{n} \beta_{n} \frac{\|F p\| \mu}{\tau}+\left(1-\tau \alpha_{n} \beta_{n}\right)\left\|x_{n}-p\right\| \\
& \leq \max \left\{\frac{\|F p\| \mu}{\tau},\left\|x_{n}-p\right\|\right\}
\end{aligned}
$$

from which we conclude that $\left\{x_{n}\right\}$ is a bounded vector sequence in $H_{1}$. 
Step 2 . We prove that $\lim _{n \rightarrow \infty}\left\|x_{n+1}-x_{n}\right\|=0$. From resolvent equality (1.6) in Lemma 1.6 we see that

$$
\begin{aligned}
\left\|z_{n}-z_{n+1}\right\| & \leq\left\|\operatorname{Res}_{s_{n}}^{N} \rho_{n}-\operatorname{Res}_{s_{n+1}}^{N} \rho_{n}\right\|+\left\|\operatorname{Res}_{s_{n+1}}^{N} \rho_{n}-\operatorname{Res}_{s_{n+1}}^{N} \rho_{n+1}\right\| \\
& \leq\left\|\operatorname{Res}_{s_{n}}^{N} \rho_{n}-\operatorname{Res}_{s_{n+1}}^{N} \rho_{n}\right\|+\left\|\rho_{n}-\rho_{n+1}\right\| \\
& =\left\|\operatorname{Res}_{s_{n+1}}^{N}\left(\frac{s_{n+1}}{s_{n}} \rho_{n}+\left(1-\frac{s_{n+1}}{s_{n}}\right) \operatorname{Res}_{s_{n}}^{N} \rho_{n}\right)-\operatorname{Res}_{s_{n+1}}^{N} \rho_{n}\right\|+\left\|\rho_{n}-\rho_{n+1}\right\| \\
& =\left\|\left(\frac{s_{n+1}}{s_{n}} \rho_{n}+\left(1-\frac{s_{n+1}}{s_{n}}\right) \operatorname{Res}_{s_{n}}^{N} \rho_{n}\right)-\rho_{n}\right\|+\left\|\rho_{n}-\rho_{n+1}\right\| \\
& \leq\left|1-\frac{s_{n+1}}{s_{n}}\right|\left\|\rho_{n}-\operatorname{Res}_{s_{n}}^{N} \rho_{n}\right\|+\left\|\rho_{n+1}-\rho_{n}\right\|,
\end{aligned}
$$

where

$$
\rho_{n}=x_{n}+\gamma A^{*}\left(\operatorname{Res}_{r_{n}}^{M}-I\right) A x_{n}
$$

It is easy to see that

$$
\begin{aligned}
& \left\|\left(x_{n+1}-x_{n}\right)-\gamma A^{*}\left(A x_{n+1}-A x_{n}\right)\right\| \\
& \quad=\sqrt{\left\|x_{n+1}-x_{n}\right\|^{2}-2 \gamma\left\langle x_{n+1}-x_{n}, A^{*}\left(A x_{n+1}-A x_{n}\right)\right\rangle+\left\|\gamma A^{*}\left(A x_{n+1}-A x_{n}\right)\right\|^{2}} \\
& \quad=\left(1-\gamma\|A\|^{2}\right)\left\|x_{n+1}-x_{n}\right\|,
\end{aligned}
$$

which sends us to

$$
\begin{aligned}
& \left\|\rho_{n+1}-\rho_{n}\right\| \\
& \quad \leq \gamma\left\|A^{*}\left(\operatorname{Res}_{r_{n+1}}^{M} A x_{n+1}-\operatorname{Res}_{r_{n}}^{M} A x_{n}\right)\right\|+\left\|\left(x_{n+1}-x_{n}\right)-\gamma A^{*}\left(A x_{n+1}-A x_{n}\right)\right\| \\
& \quad \leq\left\|x_{n+1}-x_{n}\right\|+\gamma\|A\| \mid 1-\frac{r_{n+1}}{r_{n}}\|\| A x_{n}-\operatorname{Res}_{r_{n}}^{M} A x_{n} \| .
\end{aligned}
$$

Inequalities (2.4) and (2.5) yield

$$
\begin{aligned}
\left\|z_{n}-z_{n+1}\right\| \leq & \left|1-\frac{s_{n+1}}{s_{n}}\right|\left\|\rho_{n}-\operatorname{Res}_{s_{n}}^{N} \rho_{n}\right\|+\left\|x_{n+1}-x_{n}\right\| \\
& +\gamma\|A\|\left|1-\frac{r_{n+1}}{r_{n}}\right| \mid\left\|A x_{n}-\operatorname{Res}_{r_{n}}^{M} A x_{n}\right\|,
\end{aligned}
$$

which further leads us to

$$
\begin{aligned}
\left\|y_{n}-y_{n+1}\right\| \leq & \gamma_{n}\left\|z_{n}-z_{n+1}\right\|+\left(1-\gamma_{n}\right)\left\|x_{n}-x_{n+1}\right\|+\left|\gamma_{n}-\gamma_{n+1}\right|\left\|z_{n+1}-x_{z+1}\right\| \\
\leq & \gamma_{n}\left|1-\frac{s_{n+1}}{s_{n}}\right|\left\|\rho_{n}-\operatorname{Res}_{s_{n}}^{N} \rho_{n}\right\|+\left\|x_{n+1}-x_{n}\right\| \\
& +\gamma_{n} \gamma\|A\|\left|1-\frac{r_{n+1}}{r_{n}}\right| \mid\left\|A x_{n}-\operatorname{Res}_{r_{n}}^{M} A x_{n}\right\| \\
& +\left|\gamma_{n}-\gamma_{n+1}\right|\left\|z_{n+1}-x_{z+1}\right\| .
\end{aligned}
$$


From Lemma 1.1 we arrive at

$$
\begin{aligned}
& \left\|W_{n+1} y_{n+1}-W_{n} y_{n}\right\| \\
& \leq\left\|W_{n+1} y_{n+1}-W_{n} y_{n+1}\right\|+\left\|W_{n} y_{n+1}-W_{n} y_{n}\right\| \\
& \leq \sup _{x \in \Psi}\left[\left\|W_{n+1} x-W x\right\|+\left\|W x-W_{n} x\right\|\right]+\left\|y_{n+1}-y_{n}\right\| \\
& \leq \sup _{x \in \Psi}\left[\left\|W_{n+1} x-W x\right\|+\left\|W x-W_{n} x\right\|\right]+\gamma_{n}\left|1-\frac{s_{n+1}}{s_{n}}\right|\left\|\rho_{n}-\operatorname{Res}_{s_{n}}^{N} \rho_{n}\right\| \\
& \quad+\left\|x_{n+1}-x_{n}\right\|+\gamma_{n} \gamma\|A\|\left|1-\frac{r_{n+1}}{r_{n}}\right| \mid\left\|A x_{n}-\operatorname{Res}_{r_{n}}^{M} A x_{n}\right\| \\
& \quad+\left|\gamma_{n}-\gamma_{n+1}\right|\left\|z_{n+1}-x_{z+1}\right\|,
\end{aligned}
$$

where $\Psi$ is a bounded set containing $\left\{y_{n}\right\}$. Inequality (2.6) ensures that

$$
\begin{aligned}
\|(I- & \left.\mu \alpha_{n+1} F\right) W_{n+1} y_{n+1}-\left(I-\mu \alpha_{n} F\right) W_{n} y_{n} \| \\
\leq & \left\|\left(I-\mu \alpha_{n+1} F\right) W_{n+1} y_{n+1}-\left(I-\mu \alpha_{n+1} F\right) W_{n} y_{n}\right\| \\
& +\left\|\left(I-\mu \alpha_{n+1} F\right) W_{n} y_{n}-\left(I-\mu \alpha_{n} F\right) W_{n} y_{n}\right\| \\
\leq & \left(1-\tau \alpha_{n+1}\right)\left\|W_{n+1} y_{n+1}-W_{n} y_{n}\right\|+\left|\alpha_{n+1}-\alpha_{n}\right|\left\|\mu F W_{n} y_{n}\right\| \\
\leq & \left(1-\tau \alpha_{n+1}\right)\left\|W_{n+1} y_{n+1}-W_{n} y_{n}\right\|+\left|\alpha_{n+1}-\alpha_{n}\right|\left\|\mu F W_{n} y_{n}\right\| \\
\leq & \left(1-\tau \alpha_{n+1}\right) \sup _{x \in \Psi}\left[\left\|W_{n+1} x-W x\right\|+\left\|W x-W_{n} x\right\|\right] \\
& +\left(1-\tau \alpha_{n+1}\right) \gamma_{n}\left|1-\frac{s_{n+1}}{s_{n}}\right|\left\|\rho_{n}-\operatorname{Res}_{s_{n}}^{N} \rho_{n}\right\| \\
& +\left(1-\tau \alpha_{n+1}\right)\left\|x_{n+1}-x_{n}\right\|+\left(1-\tau \alpha_{n+1}\right) \gamma_{n} \gamma\|A\|\left|1-\frac{r_{n+1}}{r_{n}}\right| \mid\left\|A x_{n}-\operatorname{Res}_{r_{n}}^{M} A x_{n}\right\| \\
& +\left(1-\tau \alpha_{n+1}\right)\left|\gamma_{n}-\gamma_{n+1}\right|\left\|z_{n+1}-x_{z+1}\right\|+\left|\alpha_{n+1}-\alpha_{n}\right|\left\|\mu F W_{n} y_{n}\right\| .
\end{aligned}
$$

This further leads to

$$
\begin{aligned}
& \left\|\left(I-\mu \alpha_{n+1} F\right) W_{n+1} y_{n+1}-\left(I-\mu \alpha_{n} F\right) W_{n} y_{n}\right\|-\left\|x_{n+1}-x_{n}\right\| \\
& \leq \sup _{x \in \Psi}\left[\left\|W_{n+1} x-W x\right\|+\left\|W x-W_{n} x\right\|\right]+\gamma_{n}\left|1-\frac{s_{n+1}}{s_{n}}\right|\left\|\rho_{n}-\operatorname{Res}_{s_{n}}^{N} \rho_{n}\right\| \\
& \quad+\gamma_{n} \gamma\|A\|\left|1-\frac{r_{n+1}}{r_{n}}\right| \mid\left\|A x_{n}-\operatorname{Res}_{r_{n}}^{M} A x_{n}\right\| \\
& \quad+\left|\gamma_{n}-\gamma_{n+1}\right|\left\|z_{n+1}-x_{z+1}\right\|+\left(\left|\alpha_{n+1}\right|+\left|\alpha_{n}\right|\right)\left\|\mu F W_{n} y_{n}\right\| .
\end{aligned}
$$

Using Lemma 1.2, the boundedness of operator $A$, and the restrictions on the parameter sequences $\left\{\alpha_{n}\right\},\left\{\gamma_{n}\right\},\left\{s_{n}\right\}$, and $\left\{r_{n}\right\}$, we obtain that

$$
\limsup _{n \rightarrow \infty}\left(\left\|\left(I-\mu \alpha_{n+1} F\right) W_{n+1} y_{n+1}-\left(I-\mu \alpha_{n} F\right) W_{n} y_{n}\right\|-\left\|x_{n+1}-x_{n}\right\|\right) \leq 0
$$


With the aid of Lemma 1.7, we conclude that

$$
\lim _{n \rightarrow \infty}\left\|\left(I-\mu \alpha_{n} F\right) W_{n} y_{n}-x_{n}\right\|=0 .
$$

Since $\alpha_{n} \rightarrow 0$ as $n \rightarrow \infty$, we also have

$$
\lim _{n \rightarrow \infty}\left\|W_{n} y_{n}-x_{n}\right\|=0 .
$$

From (2.7) we see that

$$
\lim _{n \rightarrow \infty}\left\|x_{n+1}-x_{n}\right\|=0 .
$$

Since $\left\{x_{n}\right\}$ is a bounded vector sequence in $H_{1}$, we find that there is a subsequence $\left\{x_{n_{i}}\right\}$ of $\left\{x_{n}\right\}$ that converges weakly to $\bar{x}$.

Step 3. We prove that $x \in \bigcap_{i=1}^{\infty} \operatorname{Fix}\left(S_{i}\right) \cap \operatorname{SIP}(M, N)$.

Put

$$
\varphi_{n}=\left(I-\mu \alpha_{n} F\right) W_{n} y_{n}
$$

For any $p \in \bigcap_{i=1}^{\infty} \operatorname{Fix}\left(S_{i}\right) \cap \operatorname{SIP}(M, N)$, we conclude from (2.3) that

$$
\begin{aligned}
\left\|\varphi_{n}-p\right\|^{2} \leq & \left\|\left(I-\mu \alpha_{n} F\right) W_{n} y_{n}-\left(I-\mu \alpha_{n} F\right) W_{n} p\right\|^{2}-2 \mu \alpha_{n}\left\langle\varphi_{n}-p, F p\right\rangle \\
\leq & \left(1-\tau \alpha_{n}\right)^{2}\left\|W_{n} y_{n}-W_{n} p\right\|^{2}-2 \mu \alpha_{n}\left\langle\varphi_{n}-p, F p\right\rangle \\
\leq & \left(1-\tau \alpha_{n}\right)^{2}\left\|y_{n}-p\right\|^{2}-2 \mu \alpha_{n}\left\langle\varphi_{n}-p, F p\right\rangle \\
\leq & \left(1-\tau \alpha_{n}\right)^{2}\left\|x_{n}-p\right\|^{2}-\gamma\left(1-\gamma\|A\|^{2}\right)\left(1-\tau \alpha_{n}\right)^{2}\left\|\operatorname{Res}_{r_{n}}^{M} A x_{n}-A x_{n}\right\|^{2} \\
& +2 \mu \alpha_{n}\|F p\|\left\|\varphi_{n}-p\right\| .
\end{aligned}
$$

This shows us that

$$
\begin{aligned}
\left\|x_{n+1}-p\right\|^{2} \leq & \beta_{n}\left\|\varphi_{n}-p\right\|^{2}+\left(1-\beta_{n}\right)\left\|x_{n}-p\right\|^{2} \\
\leq & \left\|x_{n}-p\right\|^{2}-\beta_{n} \gamma\left(1-\gamma\|A\|^{2}\right)\left(1-\tau \alpha_{n}\right)^{2}\left\|\operatorname{Res}_{r_{n}}^{M} A x_{n}-A x_{n}\right\|^{2} \\
& \quad+2 \mu \alpha_{n} \beta_{n}\|F p\|\left\|\varphi_{n}-p\right\| .
\end{aligned}
$$

It follows that

$$
\begin{aligned}
& \gamma\left(1-\gamma\|A\|^{2}\right)\left(1-\tau \alpha_{n}\right)^{2} \beta_{n}\left\|A x_{n}-\operatorname{Res}_{r_{n}}^{M} A x_{n}\right\|^{2} \\
& \quad \leq\left\|x_{n}-x_{n+1}\right\|\left(\left\|x_{n}-p\right\|+\left\|x_{n+1}-p\right\|\right)+2 \mu \alpha_{n} \beta_{n}\|F p\|\left\|\varphi_{n}-p\right\| .
\end{aligned}
$$

Limit (2.9) and the fact that $\alpha_{n} \rightarrow 0$ as $n \rightarrow \infty$ lead us to

$$
\lim _{n \rightarrow \infty}\left\|A x_{n}-\operatorname{Res}_{r_{n}}^{M} A x_{n}\right\|=0 .
$$

Next, we have

$$
\begin{aligned}
& 2\left\|z_{n}-p\right\|^{2} \\
& \quad \leq 2\left\langle\gamma A^{*}\left(\operatorname{Res}_{r_{n}}^{M}-I\right) A x_{n}+x_{n}-p, z_{n}-p\right\rangle
\end{aligned}
$$




$$
\begin{aligned}
= & \gamma^{2}\left\|A^{*}\left(\operatorname{Res}_{r_{n}}^{M}-I\right) A x_{n}\right\|^{2}+2 \gamma\left\langle A^{*}\left(\operatorname{Res}_{r_{n}}^{M}-I\right) A x_{n}, x_{n}-p\right\rangle+\left\|x_{n}-p\right\|^{2} \\
& -\left\|x_{n}+\gamma A^{*}\left(\operatorname{Res}_{r_{n}}^{M}-I\right) A x_{n}-y_{n}\right\|^{2}+\left\|z_{n}-p\right\|^{2} \\
\leq & \gamma^{2}\|A\|^{2}\left\|\operatorname{Res}_{r_{n}}^{M} A x_{n}-A x_{n}\right\|^{2} \\
& +2 \gamma\left(\left\langle\operatorname{Res}_{r_{n}}^{M} A x_{n}-A p, \operatorname{Res}_{r_{n}}^{M} A x_{n}-A x_{n}\right\rangle-\left\|\operatorname{Res}_{r_{n}}^{M} A x_{n}-A x_{n}\right\|^{2}\right) \\
& +\left\|z_{n}-p\right\|^{2}+\left\|x_{n}-p\right\|^{2}-\left\|z_{n}-x_{n}\right\|^{2}-2 \gamma\left\langle A^{*}\left(\operatorname{Res}_{r_{n}}^{M}-I\right) A x_{n}, x_{n}-z_{n}\right\rangle \\
& \left.-\left\|\gamma A^{*}\left(\operatorname{Res}_{r_{n}}^{M}-I\right) A x_{n}\right\|^{2}\right) \\
\leq & \left\|x_{n}-p\right\|^{2}+\left\|z_{n}-p\right\|^{2}+2\|A\| \gamma\left\|x_{n}-z_{n}\right\|\left\|\operatorname{Res}_{r_{n}}^{M} A x_{n}-A x_{n}\right\|-\left\|x_{n}-z_{n}\right\|^{2},
\end{aligned}
$$

that is,

$$
\left\|z_{n}-p\right\|^{2} \leq\left\|x_{n}-p\right\|^{2}+2\|A\| \gamma\left\|z_{n}-x_{n}\right\|\left\|\operatorname{Res}_{s_{n}}^{N} A x_{n}-A x_{n}\right\|-\left\|x_{n}-z_{n}\right\|^{2} .
$$

This sends us to

$$
\begin{aligned}
\left\|\varphi_{n}-p\right\|^{2} \leq & \left(1-\tau \alpha_{n}\right)^{2}\left\|W_{n} y_{n}-W_{n} p\right\|^{2}-2 \mu \alpha_{n}\left\langle\varphi_{n}-p, F p\right\rangle \\
\leq & \left(1-\tau \alpha_{n}\right)^{2} \gamma_{n}\left\|z_{n}-p\right\|^{2}+\left(1-\tau \alpha_{n}\right)^{2}\left(1-\gamma_{n}\right)\left\|x_{n}-p\right\|^{2}+2 \mu \alpha_{n}\left\|\varphi_{n}-p\right\|\|F p\| \\
\leq & \left(1-\tau \alpha_{n}\right)^{2}\left\|x_{n}-p\right\|^{2}+2\left(1-\tau \alpha_{n}\right)^{2} \gamma_{n}\|A\| \gamma\left\|z_{n}-x_{n}\right\|\left\|\operatorname{Res}_{s_{n}}^{N} A x_{n}-A x_{n}\right\| \\
& -\left(1-\tau \alpha_{n}\right)^{2} \gamma_{n}\left\|x_{n}-z_{n}\right\|^{2}+2 \mu \alpha_{n}\left\|\varphi_{n}-p\right\|\|F p\| .
\end{aligned}
$$

It follows that

$$
\begin{aligned}
\left\|x_{n+1}-p\right\|^{2} \leq & \beta_{n}\left\|\varphi_{n}-p\right\|^{2}+\left(1-\beta_{n}\right)\left\|x_{n}-p\right\|^{2} \\
\leq & \left\|x_{n}-p\right\|^{2}+2 \beta_{n}\left(1-\tau \alpha_{n}\right)^{2} \gamma_{n}\|A\| \gamma\left\|z_{n}-x_{n}\right\|\left\|\operatorname{Res}_{s_{n}}^{N} A x_{n}-A x_{n}\right\| \\
& -\beta_{n}\left(1-\tau \alpha_{n}\right)^{2} \gamma_{n}\left\|x_{n}-z_{n}\right\|^{2}+2 \mu \alpha_{n} \beta_{n}\left\|\varphi_{n}-p\right\|\|F p\| .
\end{aligned}
$$

Hence

$$
\begin{aligned}
& \beta_{n}\left(1-\tau \alpha_{n}\right)^{2} \gamma_{n}\left\|x_{n}-z_{n}\right\|^{2} \\
& \quad \leq\left\|x_{n}-x_{n+1}\right\|\left(\left\|x_{n}-p\right\|+\left\|x_{n+1}-p\right\|\right)+2\|A\| \gamma\left\|z_{n}-x_{n}\right\|\left\|\operatorname{Res}_{s_{n}}^{N} A x_{n}-A x_{n}\right\| \\
& \quad+2 \mu \alpha_{n}\left\|\varphi_{n}-p\right\|\|F p\| .
\end{aligned}
$$

Using (2.9) and (2.10), we have that $x_{n}-z_{n} \rightarrow 0$ as $n \rightarrow \infty$, that is,

$$
\lim _{n \rightarrow \infty}\left\|x_{n}-\operatorname{Res}_{s_{n}}^{N}\left(x_{n}+\gamma A^{*}\left(\operatorname{Res}_{r_{n}}^{M}-I\right) A x_{n}\right)\right\|=0 .
$$

Since $x_{n}-z_{n} \rightarrow 0$ as $n \rightarrow \infty$, we have that $\left\{z_{n}\right\}$ converges weakly to $\bar{x}$. Further, $\left\{z_{n_{i}}\right\}$ converges weakly to $\bar{x}$ as $i \rightarrow \infty$. The graphs of maximal monotone mappings are weaklystrongly closed. Observe that

$$
\frac{x_{n_{i}}-z_{n_{i}}}{s_{n_{i}}}+\gamma A^{*} \frac{\operatorname{Res}_{r_{n_{i}}}^{M} A x_{n_{i}}-A x_{n_{i}}}{s_{n_{i}}} \in N z_{n_{i}} .
$$


So $0 \in N(\bar{x})$. Fixing a positive real number $p$, Lemma 1.6 yields that $\left\|A x_{n_{i}}-\operatorname{Res}_{p}^{M} A x_{n_{i}}\right\| \rightarrow$ as $i \rightarrow \infty$, which implies $0 \in M(A \bar{x})$.

We are now in a position to show that $\bar{x} \in \bigcap_{i=1}^{\infty} \operatorname{Fix}\left(S_{i}\right)=\operatorname{Fix}(W)$. We have

$$
\begin{aligned}
\left\|y_{n_{i}}-W_{n_{i}} y_{n_{i}}\right\| & \leq\left\|y_{n_{i}}-W_{n_{i}} y_{n_{i}}\right\|+\left\|W_{n_{i}} y_{n_{i}}-W y_{n_{i}}\right\| \\
& \leq\left\|y_{n_{i}}-W_{n_{i}} y_{n_{i}}\right\|+\sup _{x \in \Psi}\left\|W_{n_{i}} x-W x\right\| .
\end{aligned}
$$

Relations (2.8) and (2.11) yield that $\lim _{i \rightarrow \infty}\left\|y_{n_{i}}-W_{n_{i}} y_{n_{i}}\right\|=0$. If $\bar{x} \neq W \bar{x}$, then the Opial condition, Lemma 1.5, sends us to

$$
\begin{aligned}
\limsup _{i \rightarrow \infty}\left\|\bar{x}-y_{n_{i}}\right\| & <\limsup _{i \rightarrow \infty}\left\|W \bar{x}-y_{n_{i}}\right\| \\
& \leq \limsup _{i \rightarrow \infty}\left\{\left\|W y_{n_{i}}-y_{n_{i}}\right\|+\left\|W \bar{x}-W y_{n_{i}}\right\|\right\} \\
& \leq \limsup _{i \rightarrow \infty}\left\|\bar{x}-y_{n_{i}}\right\|,
\end{aligned}
$$

a contradiction. Thus $\bar{x} \in \operatorname{Fix}(W)$, that is, $\bar{x} \in \bigcap_{i=1}^{\infty} \operatorname{Fix}\left(S_{i}\right)$.

Step 4 . We prove that the sequence $\left\{x_{n}\right\}$ is strongly convergent.

Since $F$ is strongly monotone and Lipschitz continuous, we get that the following variational inequality has a unique solution:

$$
\langle\widetilde{x}-y, \widetilde{x}\rangle \leq 0, \quad \forall y \in \bigcap_{i=1}^{\infty} \operatorname{Fix}\left(S_{i}\right) \cap \operatorname{SIP}(M, N) .
$$

Thus

$$
\limsup _{n \rightarrow \infty}\left\langle\widetilde{x}-\varphi_{n}, F \widetilde{x}\right\rangle \leq 0
$$

Lemma 1.1 and Lemma 1.3 send us to

$$
\begin{aligned}
& \left\|x_{n+1}-\tilde{x}\right\|^{2} \\
& \quad \leq \beta_{n}\left\|\varphi_{n}-\widetilde{x}\right\|^{2}+\left(1-\beta_{n}\right)\left\|x_{n}-\widetilde{x}\right\|^{2} \\
& \quad \leq \beta_{n}\left(\left\|\left(I-\mu \alpha_{n} F\right) W_{n} y_{n}-\left(I-\mu \alpha_{n} F\right) \widetilde{x}\right\|^{2}-2 \mu \alpha_{n}\left\langle\varphi_{n}-\widetilde{x}, F(\widetilde{x})\right\rangle\right)+\left(1-\beta_{n}\right)\left\|x_{n}-\widetilde{x}\right\|^{2} \\
& \quad \leq \beta_{n}\left(\left(1-\tau \alpha_{n}\right)^{2}\left\|y_{n}-\bar{x}\right\|^{2}-2 \mu \alpha_{n}\left\langle F(\widetilde{x}), \varphi_{n}-\widetilde{x}\right)\right)+\left(1-\beta_{n}\right)\left\|x_{n}-\tilde{x}\right\|^{2} \\
& \quad \leq\left(1-2 \tau \beta_{n} \alpha_{n}\right)\left\|x_{n}-\tilde{x}\right\|^{2}+2 \tau \beta_{n} \alpha_{n} \Pi_{n}
\end{aligned}
$$

where $\Pi=\frac{\mu}{\tau}\left\langle F \bar{x}, \tilde{x}-\varphi_{n}\right\rangle+\frac{\tau \alpha_{n}}{2}\left\|x_{n}-\tilde{x}\right\|^{2}$. In light of Lemma 1.4, we find that $\left\|x_{n}-\tilde{x}\right\| \rightarrow 0$ as $n \rightarrow \infty$. This completes the proof.

From Theorem 2.1 we have the following subresult on split inclusion problem (1.3).

Corollary 2.1 Let $H_{1}$ and $H_{2}$ be Hilbert spaces, and let $N$ and $M$ be set-valued maximal monotone mappings on $H_{1}$ and $H_{2}$, respectively. Let $F: H_{1} \rightarrow H_{1}$ be an $\mathcal{L}$-Lipschitz continuous and $\tau$-strongly monotone mapping. Let $A$ be a linear bounded operator from $H_{1}$ to 
$H_{2}$, and let $A^{*}$ be its the adjoint operator. Assume that $\operatorname{SIP}(M, N) \neq \emptyset$. Let $\left\{x_{n}\right\}$ be the vector sequence in $H_{1}$ generated by the iterative process

$$
x_{1} \in H_{1}, \quad x_{n+1}=\beta_{n}\left(I-\mu \alpha_{n} F\right) \operatorname{Res}_{s_{n}}^{N}\left(x_{n}+\gamma A^{*}\left(\operatorname{Res}_{r_{n}}^{M}-I\right) A x_{n}\right)+\left(1-\beta_{n}\right) x_{n}, \quad n \geq 1,
$$

where $\gamma$ and $\mu$ are two positive real numbers, $\left\{s_{n}\right\}$ and $\left\{r_{n}\right\}$ are two positive real number sequences, and $\left\{\alpha_{n}\right\}$ and $\left\{\beta_{n}\right\}$ are real number sequences in $(0,1)$. Suppose that $\gamma \in\left(0, \frac{1}{\|A\|^{2}}\right)$, $\mu \in\left(0, \frac{2 \tau}{\mathcal{L}^{2}}\right), \liminf _{n \rightarrow \infty} s_{n}>0, \lim _{n \rightarrow \infty}\left|s_{n}-s_{n+1}\right|<\infty, \liminf _{n \rightarrow \infty} r_{n}>0, \lim _{n \rightarrow \infty}\left|r_{n}-r_{n+1}\right|<$ $\infty, \sum_{n=1}^{\infty} \alpha_{n}=\infty,\left\{\beta_{n}\right\}$ is a number sequence in $\left[\bar{\beta}, \bar{\beta}^{\prime}\right]$, where $\bar{\beta}$ and $\bar{\beta}^{\prime}$ are two real numbers in $(0,1)$, such that $\lim _{n \rightarrow \infty}\left|\beta_{n+1}-\beta_{n}\right|=0$. Then the sequence $\left\{x_{n}\right\}$ converges strongly to $\tilde{x} \in$ $H_{1}$, which is a unique solution of the variational inequality $\langle\widetilde{x}-y, F \widetilde{x}\rangle \leq 0, \forall y \in \operatorname{SIP}(M, N)$.

Remark 2.1 In this paper, we investigated the descent iterative methods for split inclusion problem with a common fixed point constraint of an infinite family of nonexpansive mappings. It deserves mentioning that our method does not involve projections. A solution theorem of the problem was established in the framework of Hilbert spaces under some weak assumptions imposed on different mappings and control sequences.

Acknowledgements

The authors thank the learned referees for their comments and appreciation.

Funding

This project was funded by the Deanship of Scientific Research (DSR) at King Abdulaziz University, Jeddah, under grant no. KEP-2-130-39. Therefore the authors acknowledge with thanks DSR for technical and financial support.

Availability of data and materials

All data generated or analyzed during this study are included in this paper.

Competing interests

The authors declare that they have no competing interests.

Authors' contributions

All authors contributed equally to the manuscript and approved the final manuscript.

\section{Author details}

'Department of Mathematics, King Abdulaziz University, Jeddah, Saudi Arabia. ${ }^{2}$ General Education Center, National Yunlin University of Science and Technology, Douliou, Taiwan.

\section{Publisher's Note}

Springer Nature remains neutral with regard to jurisdictional claims in published maps and institutional affiliations.

Received: 23 April 2019 Accepted: 7 August 2019 Published online: 22 August 2019

\section{References}

1. Al-Mazrooei, A.E., Latif, A., Qin, X., Yao, Y.-C.: Fixed point algorithms for split feasibility problems. Fixed Point Theory 20, 245-254 (2019)

2. Ansari, Q.H., Babu, F., Yao, J.-C.: Regularization of proximal point algorithms in Hadamard manifolds. J. Fixed Point Theory Appl. 21, Article ID 25 (2019)

3. Ansari, Q.H., Rehan, A.: Iterative methods for generalized split feasibility problems in Banach spaces. Carpath. J. Math. 33, 9-26 (2017)

4. Ansari, Q.H., Rehan, A., Wen, C.-F.: Implicit and explicit algorithms for split common fixed point problems. J. Nonlinear Convex Anal. 17, 1381-1397 (2016)

5. Ansari, Q.H., Rehan, A., Yao, J.-C.: Split feasibility and fixed point problems for asymptotically k-strict pseudo-contractive mappings in intermediate sense. Fixed Point Theory 18, 57-68 (2017)

6. Barbu, V.: Nonlinear Semigroups and Differential Equations in Banach Spaces. Noordhoff, Amsterdam (1976)

7. Ceng, L.C., Ansari, Q.H., Yao, J.-C.: Relaxed extragradient methods for finding minimum-norm solutions of the split feasibility problem. Nonlinear Anal. 75, 2116-2125 (2012)

8. Ceng, L.C., Ansari, Q.H., Yao, J.-C.: An extragradient method for solving split feasibility and fixed point problems. Comput. Math. Appl. 64, 633-642 (2012)

9. Censor, Y., Bortfeld, T., Martin, N., Trofimov, A.: A unified approach for inversion problem in intensity-modulated radiation therapy. Phys. Med. Biol. 51, 2353-2365 (2006) 
10. Censor, Y., Elfving, T:: A multiprojection algorithm using Bregman projections in a product space. Numer. Algorithms 8, 221-239 (1994)

11. Censor, Y., Elfving, T., Kopf, N., Bortfeld, T.: The multiple-sets split feasibility problem and its applications. Inverse Probl. 21, 2071-2084 (2005)

12. Chang, S.S., Lee, H.W.J., Chan, C.K.: A new method for solving equilibrium problem fixed point problem and variational inequality problem with application to optimization. Nonlinear Anal. 70, 3307-3319 (2009)

13. Chang, S.S., Wen, C.F., Yao, J.-C.: Common zero point for a finite family of inclusion problems of accretive mappings in Banach spaces. Optimization 67, 1183-1196 (2018)

14. Chang, S.S., Wen, C.F., Yao, J.-C.: Zero point problem of accretive operators in Banach spaces. Bull. Malays. Math. Sci. Soc. 42, 105-118 (2019)

15. Chang, S.S., Yao, J.-C., Wen, C.F., Wang, L.: A modified viscosity implicit-type proximal point algorithm for monotone inclusions and asymptotically nonexpansive mappings in Hadamard spaces. J. Inequal. Appl. 2018, 235 (2018)

16. Cho, S.Y.: Generalized mixed equilibrium and fixed point problems in a Banach space. J. Nonlinear Sci. Appl. 9 , 1083-1092 (2016)

17. Cho, S.Y.: Strong convergence analysis of a hybrid algorithm for nonlinear operators in a Banach space. J. Appl. Anal. Comput. 8, 19-31 (2018)

18. Cho, S.Y.: On the convergence of a modified Mann-type algorithms in Hilbert spaces. J. Nonlinear Convex Anal. 20 , 461-469 (2019)

19. Cho, S.Y., Kang, S.M.: Approximation of common solutions of variational inequalities via strict pseudocontractions. Acta Math. Sci. 34, 1607-1618 (2012)

20. Cho, S.Y., Li, W., Kang, S.M.: Convergence analysis of an iterative algorithm for monotone operators. J. Inequal. Appl. 2013, Article ID 199 (2013)

21. Dehaish, B.A.B., Qin, X., Latif, A., Bakodah, H.O.: Weak and strong convergence of algorithms for the sum of two accretive operators with applications. J. Nonlinear Convex Anal. 16, 1321-1336 (2015)

22. Liu, L:: A hybrid steepest descent method for solving split feasibility problems involving nonexpansive mappings. J. Nonlinear Convex Anal. 20, 471-488 (2019)

23. Majee, P., Nahak, C.: On inertial proximal algorithm for split variational inclusion problems. Optimization 67, $1701-1716(2018)$

24. Moudafi, A.: On the difference of two maximal monotone operators: regularization and algorithmic approaches. Appl. Math. Comput. 202, 446-452 (2008)

25. Opial, Z:: Weak convergence of successive approximations for nonexpansive mappings. Bull. Am. Math. Soc. 73, 591-597 (1967)

26. Peng, C.: A hybrid steepest descent method for a split feasibility problem in Hilbert spaces. J. Nonlinear Convex Anal. 20, 471-488 (2019)

27. Rezapour, S., Zakeri, S.H.: Strong convergence theorems for $\delta$-inverse strongly accretive operators in Banach spaces. Appl. Set-Valued Anal. Optim. 1, 39-52 (2019)

28. Shang, M.: A descent-like method for fixed points and split conclusion problems. J. Appl. Numer. Optim. 1, 91-101 (2019)

29. Shimoji, K., Takahashi, W.: Strong convergence to common fixed points of infinite nonexpansive mappings and applications. Taiwan. J. Math. 5, 387-404 (2001)

30. Suzuki, T:: Strong convergence theorems for infinite families of nonexpansive mappings in general Banach spaces. Fixed Point Theory Appl. 2005, 685918 (2005)

31. Takahashi, W., Wen, C.F., Yao, J.-C.: The shrinking projection method for a finite family of demimetric mappings with variational inequality problems in a Hilbert space. Fixed Point Theory 19, 407-419 (2018)

32. Xue, Z., Zhou, H., Cho, Y.J.: Iterative solutions of nonlinear equations for m-accretive operators in Banach spaces. J. Nonlinear Convex Anal. 1, 313-320 (2000)

33. Yamada, Y.: The hybrid steepest-descent method for variational inequalities problems over the intersection of the fixed point sets of nonexpansive mappings. In: Butnariu, D., Censor, Y., Reich, S. (eds.) Inherently Parallel Algorithms in Feasibility and Optimization and Their Applications, pp. 473-504. North-Holland, Amsterdam (2001)

34. Yang, Y., Yuan, Q.: A hybrid descent iterative algorithm for a split inclusion problem. J. Nonlinear Funct. Anal. 2018, Article ID 42 (2018)

35. Zhao, X., Ng, K.F., Li, C., Yao, J.-C.: Linear regularity and linear convergence of projection-based methods for solving convex feasibility problems. Appl. Math. Optim. 78, 613-641 (2018)

\section{Submit your manuscript to a SpringerOpen ${ }^{\circ}$ journal and benefit from:}

- Convenient online submission

- Rigorous peer review

- Open access: articles freely available online

- High visibility within the field

- Retaining the copyright to your article

Submit your next manuscript at $\boldsymbol{~ s p r i n g e r o p e n . c o m ~}$ 